\title{
Impact of standardization of antimicrobial prophylaxis duration in pediatric cardiac surgery
}

\author{
Sundeep Bath, PharmD, ${ }^{\text {a,b }}$ Jason Lines, PA, ${ }^{\mathrm{b}}$ Ann M. Loeffler, MD, ${ }^{\mathrm{b}}$ Ashim Malhotra, PhD, ${ }^{\mathrm{a}}$ and \\ R. Brigg Turner, PharmD ${ }^{a, b}$
}

\section{ABSTRACT}

Objectives: The optimal duration of antimicrobial prophylaxis following pediatric cardiac surgery is still debated. Adult studies suggest that shorter durations are adequate, but there is a paucity of data on pediatric patients.

Methods: This quasi-experimental study reviewed the charts of patients 18 years and younger who underwent cardiac surgery from April 2011 to November 2014 at a single institution. Starting in April 2013, a protocol was implemented to limit antimicrobial prophylaxis to 48 hours following sternal closure. Two analyses were performed: (1) identification of risk factors for surgical site infections from the entire cohort, and (2) comparison of surgical site infection incidence in the pre- and postprotocol groups.

Results: In the entire cohort, delayed sternal closure (adjusted odds ratio [OR], 5.7; 95\% confidence interval [CI], 1.8-17.9) and younger age (adjusted OR, $2.1 ; 95 \%$ CI, 1.1-3.8) were associated with incidence of surgical site infection. Following the protocol change, duration of antimicrobial prophylaxis decreased from $4.2 \pm 2.7$ to $1.9 \pm 1.3$ days $(P<.0001)$. After adjusting for age and delayed sternal closure, the postprotocol group had an adjusted OR of 0.98 (95\% CI, 0.32-3.00) for occurrence of surgical site infection. Other outcomes were not altered following the protocol change.

Conclusions: Restricting antimicrobial prophylaxis to 48 hours following pediatric cardiac surgery did not increase the incidence of surgical site infection at our institution. Further study is needed to validate this finding and to identify practices that reduce surgical site infections in those with delayed sternal closure. (J Thorac Cardiovasc Surg 2016;152:1115-20)

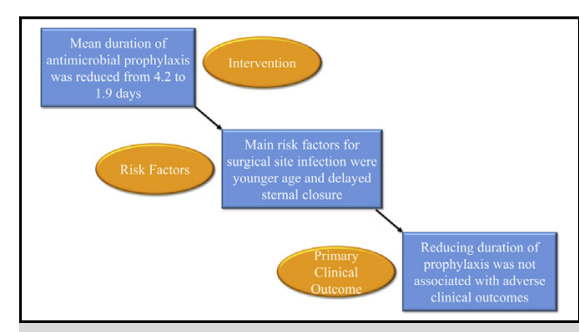

Summary of risk factors, intervention, and main result.

Central Message

Limiting antimicrobial prophylaxis to 48 hours after pediatric cardiac surgery did not increase incidence of surgical site infection.

\section{Perspective}

The optimal duration of antimicrobial prophylaxis following pediatric cardiac surgery is still debated. Limiting antimicrobial prophylaxis to 48 hours following surgery did not increase the incidence of surgical site infection. Efforts should be made to limit antimicrobial prophylaxis duration while identifying strategies to prevent infection in those patients who require delayed sternal closure.

See Editorial Commentary page 1121.
Antimicrobial prophylaxis for cardiac surgery is a widely accepted practice that has demonstrated substantial reductions in postoperative morbidity and surgical site infections (SSIs), especially for difficult to treat cardiothoracic infections such as mediastinitis and endocarditis. ${ }^{1}$ However, the optimal duration of antimicrobial prophylaxis in pediatric cardiac surgery has not been determined. Recently published consensus guidelines by the American

\footnotetext{
From the ${ }^{\mathrm{a}}$ Pacific University, School of Pharmacy, Hillsboro; and ${ }^{\mathrm{b}}$ Randall Children's Hospital at Legacy Emanuel, Portland, Ore.

Read at the 55th Interscience Conference on Antimicrobial Agents and Chemotherapy, San Diego, September 17-21, 2015.

Received for publication Jan 6, 2016; revisions received April 20, 2016; accepted for publication April 30, 2016; available ahead of print May 28, 2016.

Address for reprints: R. Brigg Turner, PharmD, Pacific University, School of Pharmacy, 222 SE 8th Ave, Suite 451, Hillsboro, OR 97123 (E-mail: brigg.turner@ pacificu.edu).

$0022-5223 / \$ 36.00$

Copyright $(2016$ by The American Association for Thoracic Surgery

http://dx.doi.org/10.1016/j.jtcvs.2016.04.091
}

Society of Health-System Pharmacists, the Infectious Diseases Society of America, the Surgical Infection Society, and the Society for Healthcare Epidemiology of America recommend antimicrobial prophylaxis to be continued up to 24 hours postoperatively in adult cardiac surgery. $^{2}$ This guideline identifies a deficit of data in pediatric patients and recommends prophylaxis in pediatrics be based on findings from adult studies. Other guidelines from the Society of Thoracic Surgeons recommend prophylaxis up to 48 hours postoperatively but also do not have formal recommendations for pediatric patients. 3,4 Basing decisions for pediatric patients on

Scanning this $\mathrm{QR}$ code will take you to the article title page.

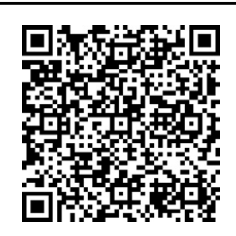




\section{Abbreviations and Acronyms \\ ICU $=$ intensive care unit \\ $\mathrm{IV}=$ intravenous \\ SSI $=$ surgical site infection}

studies comprised mainly of adults can be problematic ${ }^{5}$ because the occurrence of SSIs in pediatric cardiac procedures has been reported to be higher than in adult patients in several studies. ${ }^{6-8}$ Whereas adequate duration of antimicrobial therapy is necessary to prevent SSIs, extended prophylaxis may be associated with complications such as alteration of patient bacterial flora leading to increased colonization with resistant bacteria, superinfections including Clostridium difficile infection, and exacerbated drug toxicity. ${ }^{9}$ For this reason, selecting the shortest duration possible for antimicrobial surgical prophylaxis is preferred. ${ }^{4}$ We hypothesized that limiting antimicrobial prophylaxis postoperatively would result in a similar incidence of SSIs while also reducing overall antibiotic exposure. The purpose of this study was to evaluate the clinical impact after implementation of a protocol that limited postoperative prophylaxis to 48 hours following sternal closure in pediatric cardiac surgery.

\section{PATIENTS AND METHODS \\ Study Design}

This quasi-experimental study evaluated patients treated from April 2011 to November 2014, at Randall Children's Hospital in Portland, Oregon. Randall Children's Hospital is a 165-bed children's hospital with 24 pediatric intensive care unit (ICU) beds and a level 3 neonatal ICU with 46 beds. All patients who undergo cardiac surgery at our facility are taken immediately postoperatively to the ICU from the operating room. Incisions are protected from contamination by application of 2octyl-cyanoacrylate adhesive. In addition, bacitracin irrigation is performed prior to sternal closure in all patients. The same surgeons performed all cardiac procedures throughout the study period. Starting in April 2013, a protocol was implemented limiting antimicrobial prophylaxis to 48 hours following sternal closure; however, the decision on length of prophylaxis was ultimately at the discretion of the treating medical team. Before the protocol, the duration of antimicrobial prophylaxis was decided by each practitioner and varied substantially. This protocol was developed in response to a hospital-wide antimicrobial stewardship effort and was initiated by the cardiac surgeons and medical teams providing care for these patients with the intent to standardize care. Two separate analyses were performed. First, factors associated with incidence of SSI were identified from the entire cohort. Second, incidence of SSI was compared between the preprotocol (prior to April 2013) and postprotocol (after April 2013) groups. The Legacy Health Institutional Review Board granted a waiver of informed consent for this study.

\section{Inclusion/Exclusion Criteria}

Patients who were 18 years or younger and underwent cardiac surgery during the dates specified were screened for inclusion. Patients were excluded if they died or were discharged within 48 hours of surgery, had repeat surgery within 7 days of original surgery except for exploration for suspected infection, were receiving antimicrobials prior to surgery, or if surgery was limited to pacemaker implantation. Patients who underwent an additional surgery within 30 days of the original surgery were only included once in the study.

\section{Outcomes}

The primary outcome was incidence of SSI as defined by the Centers for Disease Control and Prevention/National Healthcare Safety Network guidelines. ${ }^{10}$ Secondary outcomes included hospital, ICU, and infectionrelated length of stay; incidence of receipt of additional antimicrobials for suspected infection; and mortality. Mortality was defined as all-cause death occurring during the admission or within 30 days of surgery.

\section{Data Collection}

Data were retrospectively collected through a hospital database which is maintained for submission of information biannually to the Congenital Heart Surgery Database of the Society of Thoracic Surgeons as well as by data extraction from the electronic health record. Appropriate prophylaxis was defined as receipt of intravenous (IV) cefazolin at a dose of $25 \mathrm{mg} / \mathrm{kg}$ or greater within 60 minutes before incision with re-dosing every 4 hours during surgery. IV vancomycin was also considered appropriate for prophylaxis for those with penicillin allergy or documented methicillinresistant Staphylococcus aureus on a preoperative nasal swab. Weight for age percentile was calculated for each patient at the time of surgery. The Society of Thoracic Surgery-European Association for Cardio-Thoracic Surgery congenital heart surgery mortality score was determined by the type of procedure being performed. ${ }^{11}$ Infection-related length of stay was calculated as the time from receipt of additional antimicrobials (not prophylaxis) until the time of discontinuation or until hospital discharge.

\section{Statistics}

Demographic and baseline data were analyzed by $t$ test, WilcoxonMann-Whitney, chi-square, and Fisher exact tests as appropriate. Univariate analysis was performed to identify factors associated with SSI in the entire cohort. Factors with $P<.2$ on univariate analysis were included in a stepwise logistic regression model with backward elimination with $P>.2$ used for elimination. Incidence of SSI and other outcomes were then compared between the pre- and postprotocol groups using $\mathrm{t}$ test, Wilcoxon-Mann-Whitney, chi-square, and Fisher exact tests as appropriate. A multivariate logistic regression model was performed to identify if the postprotocol group was associated with higher incidence of SSI after controlling for factors identified in the stepwise logistic regression model. A final model, adjusting for factors identified in the stepwise logistic regression model, was performed to identify any effect of duration of antimicrobial prophylaxis on SSI. Data with $P<.05$ were considered significant. All analyses were performed in Stata version 13 (StataCorp 2013. Stata Statistical Software: Release 13. College Station, Tex: StataCorp LP).

\section{RESULTS}

A total of 528 patients met the initial inclusion criteria and 82 were subsequently excluded. Thus, 446 patients were included in the final analysis (see Figure 1). Of these patients, almost all $(96.2 \%)$ underwent a procedure requiring sternotomy. Fifteen $(3.4 \%)$ patients developed an SSI, with 11 defined as superficial and 4 as deep incisional. Univariate analysis revealed several factors to be associated with the incidence of SSI (Table 1). Stepwise logistic regression identified younger age and delayed sternal closure to be independently associated with SSI (Table 2). The incidence of SSI in patients with delayed sternal closure was $17.6 \%$ versus $1.5 \%$ in those without delayed sternal closure $(P<.0001)$. In addition, 


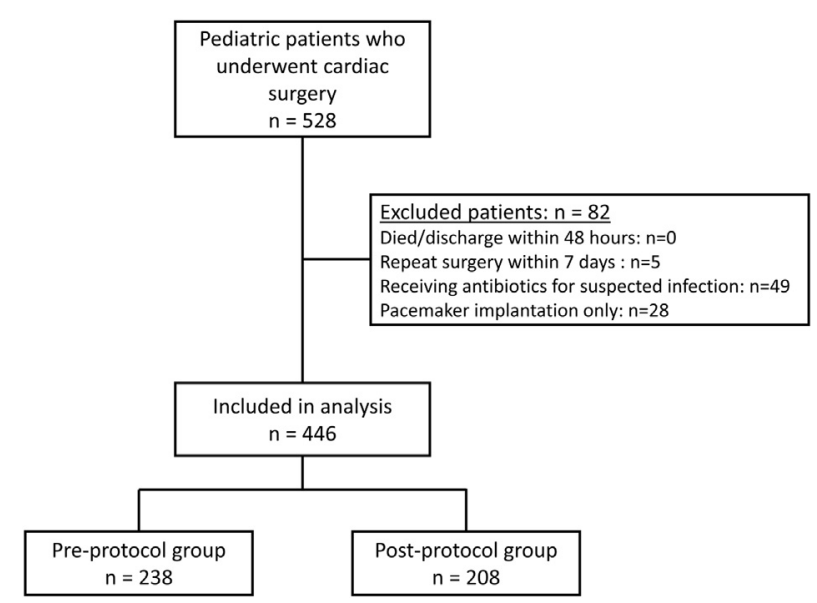

FIGURE 1. Flow diagram of patients included in the study.

all cases of SSI occurred in patients younger than 1.5 years of age.

Demographics were similar between the pre- and postprotocol groups with the exception of more frequent delivery of appropriate antimicrobial prophylaxis and lower average intraoperative glucose concentration in the

TABLE 1. Univariate analysis of risk factors for surgical site infections

\begin{tabular}{|c|c|c|c|}
\hline Risk factor & $\operatorname{SSI}(n=15)$ & $\begin{array}{c}\text { No SSI } \\
(n=431)\end{array}$ & $\begin{array}{c}P \\
\text { value }\end{array}$ \\
\hline Age $(y)$, median (IQR) & $0.17(0.0-0.27)$ & $1.7(0.25-5.8)$ & $<.0001$ \\
\hline Male gender, $\mathrm{n}(\%)$ & $10(66.7)$ & $254(58.9)$ & .61 \\
\hline Caucasian race, n (\%) & $13(86.7)$ & $345(80.0)$ & .70 \\
\hline $\begin{array}{l}\text { Percentile group of weight } \\
\text { for age }\end{array}$ & $3.2 \pm 2.4$ & $3.3 \pm 2.0$ & .87 \\
\hline STAT mortality score & $2.9 \pm 1.2$ & $2.1 \pm 1.1$ & .006 \\
\hline $\begin{array}{l}\text { Single-ventricle physiology, } \\
\text { n (\%) }\end{array}$ & $3(20.0)$ & $69(16.0)$ & .72 \\
\hline Cyanotic heart disease, $\mathrm{n}(\%)$ & $10(66.7)$ & $131(30.4)$ & .008 \\
\hline $\begin{array}{l}\text { Additional mediastinal } \\
\text { procedure, } \mathrm{n}(\%)\end{array}$ & $2(13.3)$ & $5(1.2)$ & .020 \\
\hline Delayed sternal closure, n (\%) & $9(60.0)$ & $42(9.7)$ & $<.0001$ \\
\hline Surgery duration (h) & $4.3 \pm 2.6$ & $3.8 \pm 1.8$ & .31 \\
\hline $\begin{array}{l}\text { Perioperative blood transfusion, } \\
\mathrm{n}(\%)\end{array}$ & $14(93.3)$ & $238(55.2)$ & .003 \\
\hline $\begin{array}{l}\text { Appropriate antimicrobial } \\
\text { prophylaxis, } \mathrm{n}(\%)\end{array}$ & $13(86.7)$ & $349(81.0)$ & .75 \\
\hline $\begin{array}{l}\text { Duration of antimicrobial } \\
\text { prophylaxis postclosure (d) }\end{array}$ & $3.2 \pm 2.4$ & $3.1 \pm 2.2$ & .87 \\
\hline $\begin{array}{l}\text { Intraoperative steroid } \\
\text { administration, } \mathrm{n}(\%)\end{array}$ & $1(6.7)$ & $17(3.9)$ & .47 \\
\hline $\begin{array}{l}\text { Highest intraoperative glucose } \\
\text { concentration }(\mathrm{mg} / \mathrm{dL})\end{array}$ & $205.9 \pm 40.7$ & $201.8 \pm 59.9$ & .79 \\
\hline $\begin{array}{l}\text { Cardiopulmonary bypass } \\
\text { time (min) }\end{array}$ & $151.9 \pm 133.1$ & $116.2 \pm 60.0$ & .040 \\
\hline
\end{tabular}

TABLE 2. Multivariate model of risk factors for surgical site infection from the entire study cohort

\begin{tabular}{lcc}
\hline \multicolumn{1}{c}{ Factor } & $\begin{array}{c}\text { Unadjusted odds } \\
\text { ratio }(\mathbf{9 5} \% \mathbf{C I})\end{array}$ & $\begin{array}{c}\text { Adjusted odds } \\
\text { ratio }(\mathbf{9 5} \% \mathbf{C I})\end{array}$ \\
\hline Younger age & $3.7(1.1-13.0)$ & $2.1(1.1-3.9)$ \\
Delayed sternal closure & $13.9(4.7-41.0)$ & $5.7(1.8-17.9)$ \\
\hline This analysis included risk factors with $P<.2$ on univariate analysis and eliminated
\end{tabular}
factors with $P>.2$ in the multivariate model. $C I$, Confidence interval.

postprotocol group (Table 3). Of note, there was no difference between groups in the incidence of receipt of appropriately timed preoperative antimicrobials $(94.5 \%$ vs $94.2 \%$ in the pre- and postprotocol groups, $P=.89$ ). The duration of antimicrobial prophylaxis following sternal closure decreased by greater than $50 \%$ in the postprotocol group (see Table 3). For patients without delayed sternal closure, the duration of antimicrobial prophylaxis decreased from $4.2 \pm 2.7$ days to $2.0 \pm 1.0$ days $(P<.0001)$. For those with delayed sternal closure, duration following closure decreased from $4.7 \pm 2.6$ days to $1.5 \pm 2.3$ days $(P<.0001)$, with total duration decreasing from $7.6 \pm 2.5$ days to $4.9 \pm 1.6$ days $(P<.0001)$. A total of $83 \%$ of patients in the postintervention group received prophylaxis for 48 hours or less and $89.5 \%$ received 72 hours or less of prophylaxis. It was unclear from chart review why some patients in the postprotocol group received prophylaxis for longer than 48 hours; however, duration of therapy was ultimately left to the discretion of the treating medical team.

The incidence of SSI was similar in the pre- and postprotocol groups and other outcomes also remained unchanged (Table 4). After controlling for age and delayed sternal closure, factors found to be associated with SSI in stepwise logistic regression, the postprotocol group had an adjusted odds ratio (OR) of $0.98(95 \% \mathrm{CI}, 0.32-3.00 ; P=.97)$ for occurrence of SSI. After adjusting for age and delayed sternal closure, duration of prophylaxis was not found to be associated with occurrence of SSI (adjusted OR, 1.00; 95\% CI, 0.82-1.24; $P=.96$ ). Finally, after controlling for age, delayed sternal closure, highest intraoperative glucose concentration, and receipt of appropriate prophylaxis, the postprotocol group had an adjusted OR of $0.73(95 \% \mathrm{CI}$, $0.22-2.42 ; P=.61$ ) for occurrence of SSI.

\section{DISCUSSION}

This study demonstrated that limiting antimicrobial prophylaxis to 48 hours postoperatively following pediatric cardiac surgery did not increase the incidence of SSI or alter other clinical outcomes in our cohort. In addition, duration of prophylaxis was not found to influence incidence of SSI. Multiple studies comparing single- to multidose prophylaxis and 24-hour to longer prophylaxis in adult cardiac patients identified similar incidences of SSI. ${ }^{2,12-16}$ 
TABLE 3. Demographics and risk factors in the pre- and postprotocols groups

\begin{tabular}{|c|c|c|c|}
\hline Risk factor & Preprotocol $(n=238)$ & Postprotocol $(n=208)$ & $P$ value \\
\hline Age (y), median (IQR) & $1.70(0.28-5.91)$ & $0.98(0.17-5.05)$ & .062 \\
\hline Male gender, $\mathrm{n}(\%)$ & $140(58.8)$ & $124(59.6)$ & .87 \\
\hline Caucasian race, $\mathrm{n}(\%)$ & $187(78.6)$ & $171(82.2)$ & .84 \\
\hline Percentile group of weight for age & $3.2 \pm 2.1$ & $3.4 \pm 2.1$ & .52 \\
\hline STAT mortality score & $2.1 \pm 1.1$ & $2.1 \pm 1.1$ & .43 \\
\hline Additional mediastinal procedure, $\mathrm{n}(\%)$ & $2(0.8)$ & $5(2.4)$ & .26 \\
\hline Delayed sternal closure, $\mathrm{n}(\%)$ & $22(9.2)$ & $29(13.9)$ & .12 \\
\hline Surgery duration (h) & $3.8 \pm 1.9$ & $3.8 \pm 1.6$ & .82 \\
\hline Perioperative blood transfusion, $\mathrm{n}(\%)$ & $134(56.3)$ & $118(56.7)$ & .93 \\
\hline Appropriate antimicrobial prophylaxis, $\mathrm{n}(\%)$ & $183(64.7)$ & $179(86.1)$ & .014 \\
\hline Duration of antimicrobial prophylaxis postclosure (d) & $4.2 \pm 2.7$ & $1.9 \pm 1.3$ & $<.0001$ \\
\hline Intraoperative steroid administration, $\mathrm{n}(\%)$ & $11(4.6)$ & $7(3.4)$ & .63 \\
\hline Highest intraoperative glucose concentration (mg/dL) & $209.0 \pm 63.2$ & $193.9 \pm 53.6$ & .008 \\
\hline Cardiopulmonary bypass time (min) & $117.9 \pm 63.1$ & $116.9 \pm 65.3$ & .88 \\
\hline
\end{tabular}

Data presented as mean \pm standard deviation unless otherwise noted. IQR, Interquartile range; STAT, Society of Thoracic Surgery-European Association for Cardio-Thoracic Surgery congenital heart surgery mortality score.

Controversy still exists on this topic, with some debate on whether prophylaxis for longer than 24 hours may be beneficial in cardiac surgery. ${ }^{4,17,18}$ The joint guidelines on antimicrobial prophylaxis in surgery ${ }^{2}$ recommend that pediatric efficacy be inferred from adult studies; however, this may not be ideal because of substantial differences in patient risk factors and procedures performed. ${ }^{6-8}$

To our knowledge, only one pediatric study has evaluated the efficacy of 24-hour (or less) prophylaxis to longer prophylaxis, ${ }^{3}$ whereas several studies have evaluated limiting prophylaxis to 48 hours. ${ }^{6,19}$ Knoderer and colleagues performed a single-center, retrospective study and concluded that similar infection rates existed after limiting prophylaxis to 24 hours (in comparison with approximately 4 days of prophylaxis); however, they did not report the incidence of SSI. ${ }^{3}$ Murray and colleagues performed a quasi-experimental study after developing a protocol for neonates that limited prophylaxis to 48 hours postoperatively in patients with closed sternums and 24 hours following closure in those with delayed sternal closure. ${ }^{19}$ They identified a similar incidence of SSI in the pre- and postprotocol groups and concluded that limiting

TABLE 4. Outcomes between pre- and postprotocol groups

\begin{tabular}{lccc}
\hline \multicolumn{1}{c}{ Factor } & $\begin{array}{c}\text { Preprotocol } \\
(\mathbf{n = 2 3 8})\end{array}$ & $\begin{array}{c}\text { Postprotocol } \\
(\mathbf{n = 2 0 8})\end{array}$ & $\begin{array}{c}\boldsymbol{P} \\
\text { value }\end{array}$ \\
\hline SSI & $7(2.9)$ & $8(3.8)$ & .61 \\
Median length of stay (d), n (IQR) & $7(5-12)$ & $7(5-10.5)$ & .23 \\
Median ICU length of stay (d), & $3(2-5)$ & $3(2-4)$ & .21 \\
$\quad$ n (IQR) & & & \\
Receipt of additional antimicrobials & $47(19.7)$ & $37(17.8)$ & .60 \\
$\begin{array}{l}\text { Median infection-related length } \\
\quad \text { of stay (d), n (IQR) }\end{array}$ & $11(8-15)$ & $6(4-16)$ & .14 \\
Mortality & & & \\
\hline
\end{tabular}

Data reported as number (\%) unless otherwise noted. SSI, Surgical site infection; $I Q R$, interquartile range; $I C U$, intensive care unit. prophylaxis to this duration was safe in neonates. Several other retrospective studies in pediatric cardiac surgery have found 48 hour prophylaxis to be adequate; however, these results are at high risk of bias because duration of therapy was at the complete discretion of the prescriber with no protocol described in either study ${ }^{20,21}$ In contrast, Maher and colleagues found a higher incidence of SSI after limiting prophylaxis to 48 hours in comparison with duration guided by the presence of thoracotomy tubes and vascular catheters. ${ }^{6}$ These data suggest that antimicrobial prophylaxis of 48 hours has similar efficacy to longer prophylaxis.

The current study identified a similar incidence of SSI as previous studies in pediatric cardiac surgery. ${ }^{22,23}$ Several reports have identified delayed sternal closure to be a risk factor for $\mathrm{SSI}^{6,22,24}$ but others have failed to identify this association. ${ }^{20,23}$ Differences in the outcomes of these studies may be due to other risk factors, type of surgery performed or, more likely, differences in postoperative care in patients with delayed sternal closure. As incidence of SSI was much greater in those with delayed sternal closure, we performed a post hoc analysis attempting to identify risk factors in this cohort. No association between risk factors and incidence of SSI was identified. Similar to what has been shown previously, longer duration until closure was associated with development of deep SSI (days to closure in those with and without SSI, $5.6 \pm 0.7$ days vs $2.4 \pm 0.9$ days, $P=.002) .{ }^{20}$ While it seems prudent to shorten the duration until sternal closure, sometimes this is not possible and it remains to be seen if this would result in a decreased incidence of SSI. Future efforts at Randall Children's Hospital will include evaluation of our postoperative management of patients with delayed sternal closure in hopes of reducing incidence of SSI in this population.

Our study did not support the findings of other studies that have identified duration of surgery, ${ }^{25}$ treatment with 
extracorporeal membrane oxygenation, ${ }^{22}$ excessive bleeding, ${ }^{23,26}$ and duration of cardiopulmonary bypass time $^{27,28}$ as risk factors associated with incidence of SSI. Although we did identify receipt of perioperative blood transfusions and cardiopulmonary bypass time as risk factors for SSI on univariate analysis, this did not hold true in the multivariate analysis.

Cefazolin and cefuroxime are recommended equally as first-line agents for antimicrobial prophylaxis in cardiac surgery. ${ }^{2}$ Most of our patients $(97.3 \%)$ received IV cefazolin for both pre- and postoperative prophylaxis with the remainder receiving vancomycin; this was similar in both pre- and postintervention groups. All patients who developed an SSI received cefazolin at a dose of $25-35 \mathrm{mg} / \mathrm{kg}$. The dose of cefazolin was not a predictor of SSI (mean dose in those with and without SSI, $26.7 \pm 3.1 \mathrm{mg} / \mathrm{kg}$ vs $27.2 \pm 6.8 \mathrm{mg} / \mathrm{kg} ; P=.74$ ). Of the 7 patients with SSI who had positive wound cultures, 5 were caused by organisms susceptible to cefazolin. These data suggest that a change in agent or dose is not merited at our institution.

This study had several limitations. First, this study was retrospective in design with a historical control group. Other factors changed from the pre- to postprotocol groups in addition to duration of antimicrobial prophylaxis, including lower average intraoperative glucose concentration, more frequent delivery of appropriate antimicrobial prophylaxis, numerically younger age of patients, and numerically greater number of patients with delayed sternal closure. No systematic intervention was introduced that would have resulted in a lower average intraoperative glucose concentration, and it is likely that this occurred because of more aggressive glucose control by individual anesthesiologists. Tighter control of intraoperative glucose concentration may not affect incidence of SSI, ${ }^{29}$ and we failed to identify average glucose concentration and receipt of appropriate antimicrobial prophylaxis as affecting incidence of SSI. While we attempted to control for these and other potentially confounding factors via multivariate logistic regression, residual confounding may be present. Second, determination of the primary outcome of SSI was made solely on information available in the electronic medical record. Failure to document information correctly in the electronic medical record may have led to underreporting of SSI incidence. However, this is an inherent limitation with a retrospective study and we expect the number of possible discrepancies to be similar in both pre- and postintervention groups. Third, in the postintervention group, $17 \%$ of patients received prophylaxis for longer than 48 hours. We attempted to control for this by performing an analysis evaluating duration of prophylaxis in the entire study cohort and found no association with incidence of SSI. In addition, excluding these patients from analyses did not alter the primary or secondary

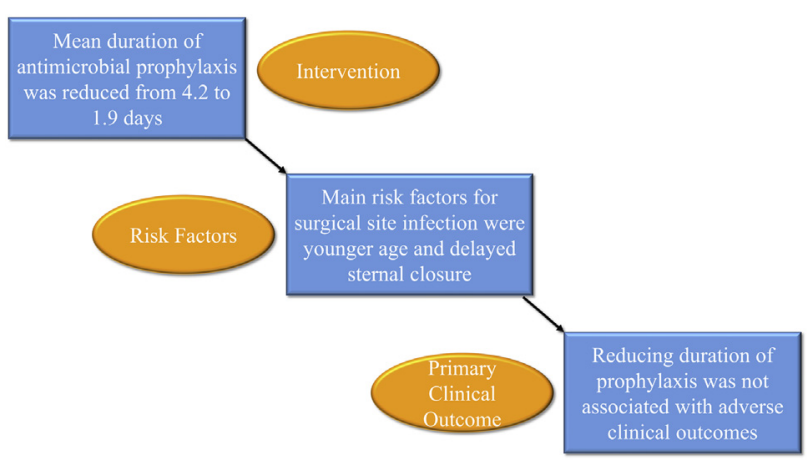

FIGURE 2. Summary of risk factors, intervention, and main result.

outcomes. Finally, this single-center, tertiary-care community hospital does not perform cardiac transplants and is in a geographic area with low rates of carriage of and infection with multidrug-resistant organisms. In addition, particular practices at our institution during and after surgery may differ from practices at other hospitals. Therefore, extrapolation of our data to other health systems should be made with caution.

Shortening the mean duration of antimicrobial prophylaxis for pediatric cardiac surgery from over 4 days to less than 48 hours following sternal closure did not increase the incidence of SSI in our cohort (Figure 2). In addition, longer duration of antimicrobial prophylaxis was not associated with decreased incidence of SSI. Limiting antimicrobial prophylaxis did not adversely affect other clinical outcomes including length of stay and mortality. This study identified younger age and delayed sternal closure to be associated with incidence of SSI. A well-powered, randomized, multicenter, controlled trial is required to verify that shorter durations of antimicrobial prophylaxis are adequate to prevent SSIs in this population. Further study is also needed in pediatric patients with delayed sternal closure to identify practices that reduce SSIs.

\section{Conflict of Interest Statement}

Authors have nothing to disclose with regard to commercial support.

We would like to acknowledge the antimicrobial stewardship pilot team at Randall Children's Hospital, the cardiology and pediatric ICU teams, and the cardiothoracic surgery team.

\section{References}

1. Kreter B, Woods M. Antibiotic prophylaxis for cardiothoracic operations. Meta-analysis of thirty years of clinical trials. J Thorac Cardiovasc Surg. 1992;104:590-9.

2. Bratzler DW, Dellinger EP, Olsen KM, Perl TM, Auwaerter PG, Bolon MK, et al. Clinical practice guidelines for antimicrobial prophylaxis in surgery. Am J Health Syst Pharm. 2013;70:195-283.

3. Knoderer CA, Cox EG, Berg MD, Webster AH, Turrentine MW. Efficacy of limited cefuroxime prophylaxis in pediatric patients after cardiovascular surgery. Am J Health Syst Pharm. 2011;68:909-14. 
4. Edwards FH, Engelman RM, Houck P, Shahian DM, Bridges CR. The Society of Thoracic Surgeons Practice Guideline Series: Antibiotic Prophylaxis in Cardiac Surgery, Part I: Duration. Ann Thorac Surg. 2006;81:397-404.

5. Klassen TP, Hartling L, Craig JC, Offringa M. Children are not just small adults: the urgent need for high-quality trial evidence in children. PLoS Med. 2008;5: e172.

6. Maher KO, Van Der Elzen K, Bove EL, Mosca RS, Chenoweth CE, Kulik TJ. A retrospective review of three antibiotic prophylaxis regimens for pediatric cardiac surgical patients. Ann Thorac Surg. 2002;74:1195-200.

7. Nateghian A, Taylor G, Robinson JL. Risk factors for surgical site infections following open-heart surgery in a Canadian pediatric population. Am J Infect Control. 2004;32:397-401.

8. Huddleston CB. Mediastinal wound infections following pediatric cardiac surgery. Semin Thorac Cardiovasc Surg. 2004;16:108-12.

9. Fry DE. Surgical site infections and the surgical care improvement project (SCIP): evolution of national quality measures. Surg Infect. 2008;9:579-84.

10. Horan TC, Andrus M, Dudeck MA. CDC/NHSN surveillance definition of health care-associated infection and criteria for specific types of infections in the acute care setting. Am J Infect Control. 2008;36:309-32.

11. O'Brien SM, Clarke DR, Jacobs JP, Jacobs ML, Lacour-Gayet FG, Pizarro C, et al. An empirically based tool for analyzing mortality associated with congenital heart surgery. J Thorac Cardiovasc Surg. 2009;138:1139-53.

12. Galbraith U, Schilling J, von Segesser LK, Carrel T, Turina M, Geroulanos S. Antibiotic prophylaxis in cardiovascular surgery: a prospective randomized comparative trial of one day cefazolin versus single dose cefuroxime. Drugs Exp Clin Res. 1993;19:229-34.

13. Kriaras I, Michalopoulos A, Michalis A, Palatianos G, Economopoulos G, Anagnostopoulos C, et al. Antibiotic prophylaxis in cardiac surgery. J Cardiovasc Surg. 1997;38:605-10.

14. Kriaras I, Michalopoulos A, Turina M, Geroulanos S. Evolution of antimicrobial prophylaxis in cardiovascular surgery. Eur J Cardiothorac Surg. 2000;18:440-6.

15. Sisto T, Laurikka J, Tarkka MR. Ceftriaxone vs cefuroxime for infection prophylaxis in coronary bypass surgery. Scand J Thorac Cardiovasc Surg. 1994;28:143-8.

16. Nooyen SM, Overbeek BP, Brutel de la Rivière A, Storm AJ, Langemeyer JJ. Prospective randomised comparison of single-dose versus multiple-dose cefuroxime for prophylaxis in coronary artery bypass grafting. Eur J Clin Microbiol Infect Dis. 1994;13:1033-7.

17. Tamayo E, Gualis J, Flórez S, Castrodeza J, Eiros Bouza JM, Alvarez FJ. Comparative study of single-dose and 24-hour multiple-dose antibiotic prophylaxis for cardiac surgery. J Thorac Cardiovasc Surg. 2008;136:1522-7.
18. Mertz D, Johnstone J, Loeb M. Does duration of perioperative antibiotic prophylaxis matter in cardiac surgery? A systematic review and meta-analysis. Ann Surg. 2011;254:48-54.

19. Murray MT, Corda R, Turcotte R, Bacha E, Saiman L, Krishnamurthy G. Implementing a standardized perioperative antibiotic prophylaxis protocol for neonates undergoing cardiac surgery. Ann Thorac Surg. 2014;98:927-33.

20. Turcotte RF, Brozovich A, Corda R, Demmer RT, Biagas KV, Mangino D, et al. Health care-associated infections in children after cardiac surgery. Pediatr Cardiol. 2014;35:1448-55.

21. Alvarez P, Fuentes C, Garcia N, Modesto V. Evaluation of the duration of the antibiotic prophylaxis in paediatric postoperative heart surgery patients. Pediatr Cardiol. 2012;33:735-8.

22. Harder E, Gaises M, Yu S, Donohue J, Haunauer D, Goldberg C, et al. Risk factors for surgical site infection in pediatric cardiac surgery patients. J Thorac Cardiovasc Surg. 2013;146:326-33

23. Murray MT, Krishnamurthy G, Corda R, Turcotte RF, Jia H, Bacha E, et al. Surgical site infections and bloodstream infections in infants after cardiac surgery. J Thorac Cardiovasc Surg. 2014;148:259-65.

24. Shah GS, Christensen RE, Wagner DS, Pearce BK, Sweeney J, Tait AR. Retrospective evaluation of antimicrobial prophylaxis in prevention of surgical site infection in the pediatric population. Paediatr Anaesth. 2014;24: 994-8.

25. Holzmann-Pazgal G, Hopkins-Broyles D, Recktenwald A, Hohrein M, Kieffer P, Huddleston C, et al. Case-control study of pediatric cardiothoracic surgical site infections. Infect Control Hosp Epidemiol. 2008;29:76-9.

26. Mohnle P, Snyder-Ramos SA, Miao Y, Kulier A, Böottiger BW, Levin J, et al. Postoperative red blood cell transfusion and morbid outcome in uncomplicated cardiac surgery patients. Intensive Care Med. 2011;37:97-109.

27. Mehta PA, Cunningham CK, Colella CB, Alferis G, Weiner LB. Risk factors for sternal wound and other infections in pediatric cardiac surgery patients. Pediatr Infect Dis J. 2000;19:1000-4.

28. Costello JM, Graham DA, Morrow DF, Morrow J, Potter-Bynoe G, Sandora TJ, et al. Risk factors for surgical site infection after cardiac surgery in children. Ann Thorac Surg. 2010;89:1833-42.

29. Agus MS, Steil GM, Wypij D, Costello JM, Laussen PC, Langer M, et al. Tight glycemic control versus standard care after pediatric cardiac surgery. $N$ Engl J Med. 2012;367:1208-19.

Key Words: antibiotics, antifungals, antiviral agents, infection, wound infection 